\title{
Theoretical perspective on strangeness production
}

\author{
Che Ming $\mathrm{Ko}^{1, \star}$ \\ ${ }^{1}$ Cyclotron Institute and Department of Physics, Texas A\&M University, College Station, Texas 77843- \\ 3366, USA
}

\begin{abstract}
A brief review of some highlights and puzzles on strangeness production in heavy ion collisions is given. These include strangeness production and the nuclear equation of state; deeply subthreshold strangeness production; mean-field potentials on strange hadrons; phi meson in dense matter; anomalous strange hadron to pion ratios; density fluctuations on particle production; $\Lambda$ hyperon polarization and the vorticity field, and exotic hadrons.
\end{abstract}

\section{Introduction}

Strangeness production in heavy ion collisions has been a topic of continuous interest since the first experiment in 1982 at Bevalac to study kaon production in various collision systems at 2.1 AGeV [1]. The motivation at that time was to use kaon as a probe of the dense matter produced in high energy heavy ion collisions because it has a much smaller scattering cross section with nucleon than the pion. Based on a simple row-on-row cascade model, the kaon yield was calculated in Ref. [2] using as input the differential cross sections for kaon production in elementary baryon-baryon collsions estimated in a simple model, and the predicted result was later found to be comparable to that measured in Ref. [1]. This was in contrast with the result from a thermal model study [3], which assumes that kaons are produced from a thermally and chemically equilibrated fireball. In this study, the predicted kaon yield was about a factor of 40 larger than that from Ref. [2], although the thermal model could describe the measured pion yield. The failure of the thermal model used in Ref. [3] was later known to be due to the overlook of exact strangeness conservation in its grand canonical treatment of particle distributions [4]. Including strangeness conservation through the canonical treatment, which is naturally included in the cascade model study of Ref. [2] through the pair production of kaon and lambda, would give a significantly smaller number of strange hadrons if their mean number is small, called the canonical suppression. It was later shown in Ref. [5] via the kinetic theory for kaon production in heavy ion collisions at the Bevalac energy that taking into account strangeness conservation would lead to the same equilibrium number as that from the canonical formulation of the conservation law in the thermal model.

With increasing collision energy than that available at Bevalac, a quark-gluon plasma (QGP) would be produced during the early stage of heavy ion collisions. Since the mass

\footnotetext{
^e-mail: ko@comp.tamu.edu
} 
of strange quark is below the temperature of the QGP, strange quarks are expected to be abundantly produced in QGP and reach chemical equilibrium with light quarks as shown in Ref. [6]. In heavy ion collisions, these strange quarks will be converted to strange hadrons, leading to an enhancement of their production than the case without the formation of a QGP. Enhanced production of strange hadrons, such as the phi meson [7], in heavy ion collisions has thus been considered as a signal for the formation of the QGP.

Because of the unique feature of strangeness production in heavy ion collisions, strange hadron production has since been studied at SIS, AGS, SPS, RHIC and LHC. The present talk will review some highlights from these studies and discuss a few puzzles that remain to be explained. These include strangeness production and the nuclear equation of state, deeply subthreshold strangeness production and baryon resonances, mean-field potentials on strange hadrons, phi meson in dense matter, anomalous strange hadron to pion ratios, density fluctuations on particle production, $\Lambda$ hyperon polarization and the vorticity field, and exotic hadrons.

\section{Strangeness production and the nuclear equation of state}

Determining the nuclear equation of state, which is important for understanding the properties of neutron stars, has been one of the main motivations for studying heavy ion collisions, in which nuclear density of many times of that in normal nuclei can be reached. Besides the collective flow of charged particles [8], the production of kaon in heavy ion collisions at energy below its production threshold in a nucleon-nucleon collision, which is $1.56 \mathrm{GeV}$, was shown in Ref. [9] to be sensitive to the stiffness of nuclear equation of state at high density. For central $\mathrm{Nb}+\mathrm{Nb}$ collisions at $700 \mathrm{AMeV}$ studied in Ref. [9], the kaon production probability from a soft nuclear equation of state with the compressibility of $200 \mathrm{MeV}$ was a factor of 2.5 larger than that from a stiff equation of state with the compressibility of $380 \mathrm{MeV}$. In both cases, the kaon is mostly produced at the compressional stage of the collision when the density is about three times that of normal nuclear matter. Comparing results from the transport model study with the experimental data from SIS for $\mathrm{Au}+\mathrm{Au}$ collisions has led to the conclusion that the nuclear equation of state is soft, similar to the conclusion from the study of the collective flow of charged particles [8].

Subthreshold strange hadron production is also sensitive to the density dependence of the nuclear symmetry energy, which is the energy needed per nucleon to convert a symmetric nuclear matter to a pure neutron matter and is essentially unknown at densities above the normal nuclear matter density [10]. Studies based on isospin-dependent transport models have shown that besides the $\pi^{-} / \pi^{-}$ratio [11], the $K^{+} / K^{0}[12,13]$ and the $\Sigma^{-} / \Sigma^{+}[14]$ ratio in heavy ion collisions with neutron-rich nuclei depend on the stiffness of the nuclear symmetry energy at high density, with the soft one giving larger values than the stiff one. Measuring these ratios of strange hadrons thus provides a potential probe to the nuclear symmetry energy at high density.

\section{Deeply subthreshold strangeness production and baryon resonances}

Strange hadron production in heavy ion collisions at subthreshold energies further provides the possibility to find the signals for the missing baryon resonances predicted by the quark model and the decay channels of known resonances that are not yet observed in experiments. A recent study of $\Xi^{-}$production using the UrQMD model [15] for $\mathrm{Ar}+\mathrm{KCl}$ collisions at 
1.76 AGeV at SIS, which is substantially below the threshold energy of $3.74 \mathrm{GeV}$ in the nucleon-nucleon collision, has shown that to describe the experimental data from the HADES Collaboration [16] requires contributions not only from the strange-exchange reactions $\bar{K} Y \rightarrow$ $\pi \Xi[17]$ and $Y Y \rightarrow N \Xi[18]$ but also from decays of $N^{*}(1990), N^{*}(2080), N^{*}(2190), N^{*}(2220)$, and $N^{*}(2250)$ to $\Xi K K$ with a branching ratio of $10 \%$, which is obtained from fitting the experimental data on $\Xi^{-}$production in nucleon-nucleon collisions. This is very different from $\Xi^{-}$production in $\mathrm{Au}+\mathrm{Au}$ collisions at $6 \mathrm{AGeV}$ at AGS, which is much above the threshold energy. In this case, the experimental data can be satisfactorily described by the transport model that includes only the reaction $\bar{K} Y \rightarrow \pi \Xi$ [19]. Including contributions from the decay of above baryon resonances to $N \phi$ with a branching ratio of $0.2 \%$, which is also determined from fitting $\phi$ meson production in nucleon-nucleon collisions, can also improve the description of the UrQMD for $\phi$ meson production in $\mathrm{Ar}+\mathrm{KCl}$ collisions at $1.76 \mathrm{AGeV}$ at SIS [15].

\section{Phi meson in nuclear medium}

The $\phi$ meson is detected in experiments through its decay either to $K \bar{K}$ or $e^{+} e^{-}\left(\mu^{+} \mu^{-}\right)$. While the former allows the determination of the number of phi mesons at the kinetic freezeout in a heavy ion collision, the latter can further provide information on the phi meson number during the expansion of the hot dense hadronic matter [20, 21]. Both earlier [22, 23] and recent [24] calculations have indicated that the phi meson absorption cross sections by the nucleon, pion and kaon are appreciable, which is consistent with the experimental results that phi meson production from nuclei is suppressed due to absorption in the nuclear medium [2527]. Since experiments from both SPS [28] and RHIC [29] have shown that the phi meson yields determined from $K \bar{K}$ and $e^{+} e^{-}\left(\mu^{+} \mu^{-}\right)$channel are similar, the phi meson number thus does not vary much during the hadronic evolution. The appreciable absorption and regeneration of phi mesons in the hadronic stage then indicates that they flow with the matter. The observation that the elliptic flow of phi mesons at RHIC follows approximately the number-of-constituent quark scaling (NCQ) by the STAR Collaboration [30] is thus consistent with the picture that the elliptic flow in heavy ion collisions at RHIC is largely produced during the partonic phase [31]. The observed violation of NCQ for the phi meson flow in heavy ion collisions at LHC by the ALICE Collaboration [32] is then the result of their scattering during the longer hadronic phase than at RHIC [33].

Although phi meson production in heavy ion collisions has been generally understood, recent experimental data from $\mathrm{Pb}+\mathrm{Pb}$ collisions at SPS show an anomalous energy dependence of the width of its rapidity distribution compared to those of other hadrons [34]. The values of the root-mean-squared rapidity width of $\pi^{-}, K^{+}, K^{-}$, and $\Lambda$ at a given collision energy decrease with increasing hadron mass, and all increase linearly with the collision energy. Both the larger width for lighter hadrons and the increase of width with collision energy are expected. The larger $K^{+}$than $K^{-}$rapidity width may be due to their respective repulsive and attractive potentials in baryon-rich matter produced at these collision energies. The anomalous behavior of the phi meson rapidity width, which is similar to that of $\Lambda$ at low collision energy but that of $\pi^{-}$at high collision energy, is at present not understood.

\section{Mean-field potentials on strange hadrons}

Effects of mean-field potentials on strange hadrons have been well studied in heavy ion collisions at SIS energies [35, 36]. In particular, the kaon and antikaon potentials at normal 
nuclear matter density are about $20 \mathrm{MeV}$ and $-120 \mathrm{MeV}$, respectively, based on the chiral effective Lagrangian that fits the empirical data on kaon- and antikaon-nucleus scattering [35]. It was shown in Ref. [37] that the repulsive kaon potential would lead to a smaller direct flow of kaons relative to that of nucleons. This effect was indeed seen in experiments at both SIS [38] and AGS [39]. The kaon direct flow almost vanishes in collisions at SIS energy and even becomes negative in collisions at AGS due to the much higher baryon density reached at higher collision energy. The repulsive kaon potential may provide an explanation for the decreasing net kaon directed flow with deceasing collision energy observed in the preliminary data from the STAR Collaboration in the beam energy scan (BES) program at RHIC. The kaon and anitkaon mean-field potentials have also been shown to contribute to the splitting between the kaon and antikaon elliptic flows in $\mathrm{Au}+\mathrm{Au}$ collisions [40, 41], which is found by the STAR Collaboration to be appreciable at $\sqrt{s_{N N}}=7.7 \mathrm{GeV}$ and decreases with increasing collision energy [42].

\section{Anomalous strange hadron to pion ratios}

One anomalous structure in the collision energy dependence of the strange hadron to pion ratios, such as the $K^{+} / \pi^{+}$and $\left(\Lambda+\Sigma^{0}\right) / \pi^{+}$, measured from heavy ion experiments at AGS, SPS and RHIC is the peak at $\sqrt{s_{N N}} \approx 8 \mathrm{GeV}$, called the horn [43]. In the statistical model description of heavy ion collisions, this has been attributed to the onset of QGP formation [43]. A recent study based on the PHSD model [44] has shown, however, that it is due to the restoration of the chiral symmetry, which leads to an enhanced production of strange quark pairs than light quark pairs from the string fragmentation during the initial stage of the collisions. It is assumed in this study that the chiral symmetry restoration proceeds before the deconfinement transition in baryon-rich matter. Such a scenario has been suggested in Ref. [45] from arguments based on the large $N_{C}$ limit of QCD. In the absence of lattice QCD calculations at finite baryon chemical potential, it is not known if nature behaves this way.

\section{Density fluctuations on strange hadron production}

The search for the critical endpoint (CEP), where the first-order phase transition from the QGP to the hadronic matter ends, of the QCD phase diagram in heavy ion collisions is currently of great interest. Studies based on both the hydrodynamic approach [46] and the microscopic transport model [47] have shown that the spinodal instability during the firstorder phase transition between the QGP and hadronic matter can induce large baryon density fluctuations. This large density fluctuation can be probed by studying ratios of particle yields. Similar to the derivation in Ref. [48] based on the coalescence model for light nuclei production, which shows that the yield ratio $\frac{N_{3_{\mathrm{H}}} N_{p}}{N_{\mathrm{d}}^{2}}$ in heavy-ion collisions is sensitive to the neutron relative density fluctuation $\Delta n=\left\langle(\delta n)^{2}\right\rangle /\langle n\rangle^{2}$ at kinetic freeze-out, the yield ratio $\frac{\Xi^{-} K^{+}}{\Lambda \phi}$ is sensitive to the $s$ quark relative density fluctuation $\Delta s=\left\langle(\delta s)^{2}\right\rangle /\langle s\rangle^{2}$ at the QGP to hadronic matter phase transition if they are produced through quark coalescence. It is known from the success of the statistical model in describing the yield ratios of hadrons that chemical freeze-out in heavy ion collisions occurs at the phase transition temperature, which has recently been shown to be related to the constancy of the entropy per particle during evolution from the chemical to the kinetic freeze-out [49]. Therefore, the yield ratios of hadrons are not expected to be modified during the hadronic evolution, and studying their dependence on collisions energy could provide a unique probe to the fluctuations of quark 
densities during the first-order phase transition from the QGP to the hadronic matter, which would help locate the CEP in the QCD phase diagram.

\section{$8 \Lambda$ hyperon polarization and the vorticity field}

Recently, the STAR Collaboration has observed that the $\Lambda$ hyperon produced in non-central relativistic heavy ion collisions is partially polarized along the direction perpendicular to the reaction plane [50]. This confirms an earlier suggestion in Ref. [51] that due to their spin-orbit interactions, quarks and antiquarks in the produced QGP can be polarized by the large orbital angular momentum in non-central heavy ion collisions, and they then lead to the production of polarized $\Lambda$ hyperons after hadronization. In more recent studies based on the hydrodynamic [52-55] and transport [56] model, the measured spin polarization of $\Lambda$ hyperons can be understood by assuming that $\Lambda$ hyperons are in thermal equilibrium in the final rotating fireball. Also, based on the chiral kinetic approach using initial conditions from a multiphase transport model [57], the spin polarizations of quarks and antiquarks in non-central heavy ion collisions have been studied in Ref. [58]. Because of the non-vanishing vorticity field due to the large orbital angular momentum in these collisions, quarks and antiquarks are found to acquire appreciable spin polarizations in the direction perpendicular to the reaction plane. Converting quarks and antiquarks to hadrons via the coalescence model, the resulting spin polarizations of $\Lambda$ and $\bar{\Lambda}$ hyperons, which are the same in this study, are comparable to those measured in experiments by the STAR Collaboration. To explain the seemingly larger $\bar{\Lambda}$ than $\Lambda$ polarization seen in the experimental data requires, however, additional mechanisms such as the magnetic field. Also, the effect of hadronic evolution on $\Lambda$ and $\bar{\Lambda}$ polarizations is not included in this study, which may also contribute to the difference in the $\Lambda$ and $\bar{\Lambda}$ polarizations because of their different dynamics in baryon-rich matter.

\section{Exotic strange hadrons}

Because of the abundant number of strange quarks present in the produced QGP, relativistic heavy ion collisions provides the opportunity to study exotic strange hadrons [59-61]. For the most known $\mathrm{H}$ dibaryon (uuddss) [62], which has the same quark structure as two $\Lambda$ hyperons, analyses of measured $\Lambda \Lambda$ correlation functions in heavy ion collisions at RHIC seem to indicate that the attractive interaction between two $\Lambda$ is not strong enough to lead to a bound state $[63,64]$. This conclusion is consistent with results from the lattice QCD calculations. Although lattice calculations show that the $\mathrm{H}$ dibaryon is bound in the massive pion cases [65], it evolves to a resonance near the $\Xi N$ threshold when the baryon-baryon potentials obtained from the lattice QCD calculations with a smaller pion mass are used in calculations based on the coupled-channel Schrödinger equation [66]. On the other hand, various theoretical models have indicated that $\Xi^{-} \Xi^{0}$ might be bound [67]. Even the diomega $\Omega \Omega$ has been shown in the chiral quark model to be bound [68], which seems to be supported by the strong attractive $\Omega \Omega$ potential from the latest lattice QCD calculation [69]. Experimental measurements of $\Xi^{-} \Xi^{0}$ and $\Omega \Omega$ correlations, as well as others such as the $p \Omega[70,71]$ and $p \Xi^{-}$correlations [72], will be of great interest to confirm these predictions

\section{Summary}

Since the first experiment at Bevalac on kaon production from heavy ion collisions and the theoretical study using a simple cascade model, a lot of progress has been made in the study 
of strangeness production in heavy ion collisions from energies below the kaon production threshold in nucleon-nucleon collisions to ultrarelativistic energies at the LHC. These studies have led to a better understanding of the nuclear equation of state at high density and the in-medium properties of strange hadrons. They also provide the possible evidence for some missing baryons resonances and their decay channels, the restoration of chiral symmetry in dense nuclear matter, and the large vorticity field in the produced QGP. The study of strange particle production in heavy ion collisions further provides the possibility to probe the QCD phase diagram through the effect of quark density fluctuations on the hadron yield ratios. However, there remain many puzzles such as the abnormal behavior in the collision energy dependence of the phi meson rapidity width, the relation between the chiral and deconfinement transition of baryon-rich QGP, and the origin of different $\Lambda$ and $\bar{\Lambda}$ polarizations. Many more progresses and puzzles, which are not discussed here, have made the study of strangeness production in heavy ion collisions one of the most fascinating topics in high energy nuclear physics. More surprises are expected in future experiments.

\section{ACKNOWLEDGEMENTS}

The author would like to thank Christoph Blume, Daniel Cebra, André Mischke and Herbert Stroebele for helpful communications and suggestions. This work was supported in part by the US Department of Energy under Contract No. DE-SC0015266 and the Welch Foundation under Grant No. A-1358.

\section{References}

[1] S. Schnetzer, M.C. Lemaire, R. Lombard, E. Moller, S. Nagamiya, G. Shapiro, H. Steiner, I. Tanihata, Phys. Rev. Lett. 49, 989 (1982)

[2] J. Randrup, C.M. Ko, Nucl. Phys. A343, 519 (1980), [Addendum: Nucl. Phys.A411,537(1983)]

[3] F. Asai, H. Sato, M. Sano, Phys. Lett. 98B, 19 (1981)

[4] R. Hagedorn, K. Redlich, Z. Phys. C27, 541 (1985)

[5] C.M. Ko, V. Koch, Z.w. Lin, K. Redlich, M.A. Stephanov, X.N. Wang, Phys. Rev. Lett. 86, $5438(2001)$

[6] J. Rafelski, B. Muller, Phys. Rev. Lett. 48, 1066 (1982), [Erratum: Phys. Rev. Lett.56,2334(1986)]

[7] A. Shor, Phys. Rev. Lett. 54, 1122 (1985)

[8] P. Danielewicz, R. Lacey, W.G. Lynch, Science 298, 1592 (2002)

[9] J. Aichelin, C.M. Ko, Phys. Rev. Lett. 55, 2661 (1985)

[10] B.A. Li, L.W. Chen, C.M. Ko, Phys. Rept. 464, 113 (2008)

[11] B.A. Li, Phys. Rev. Lett. 88, 192701 (2002)

[12] Q.f. Li, Z.x. Li, S. Soff, R.K. Gupta, M. Bleicher, H. Stoecker, J. Phys. G31, 1359 (2005)

[13] X. Lopez et al. (FOPI), Phys. Rev. C75, 011901 (2007)

[14] Q.f. Li, Z.x. Li, E.g. Zhao, R.K. Gupta, Phys. Rev. C71, 054907 (2005)

[15] J. Steinheimer, M. Bleicher, J. Phys. G43, 015104 (2016)

[16] G. Agakishiev et al., Phys. Rev. Lett. 114, 212301 (2015)

[17] C.H. Li, C.M. Ko, Nucl. Phys. A712, 110 (2002)

[18] F. Li, L.W. Chen, C.M. Ko, S.H. Lee, Phys. Rev. C85, 064902 (2012) 
[19] S. Pal, C.M. Ko, J.M. Alexander, P. Chung, R.A. Lacey, Phys. Lett. B595, 158 (2004)

[20] W.S. Chung, C.M. Ko, G.Q. Li, Nucl. Phys. A641, 357 (1998)

[21] S. Pal, C.M. Ko, Z.w. Lin, Nucl. Phys. A707, 525 (2002)

[22] W.S. Chung, G.Q. Li, C.M. Ko, Nucl. Phys. A625, 347 (1997)

[23] L. Alvarez-Ruso, V. Koch, Phys. Rev. C65, 054901 (2002)

[24] D. Cabrera, A.N. Hiller Blin, M.J. Vicente Vacas, P. Fernandez De Cordoba, Phys. Rev. C96, 034618 (2017)

[25] T. Ishikawa et al., Phys. Lett. B608, 215 (2005)

[26] M.H. Wood et al. (CLAS), Phys. Rev. Lett. 105, 112301 (2010)

[27] A. Polyanskiy et al., Phys. Lett. B695, 74 (2011)

[28] D. Adamova et al. (CERES), Phys. Rev. Lett. 96, 152301 (2006)

[29] M. Wada (STAR), Nucl. Phys. A904-905, 1019c (2013)

[30] B.I. Abelev et al. (STAR), Phys. Rev. Lett. 99, 112301 (2007)

[31] J.H. Chen, Y.G. Ma, G.L. Ma, X.Z. Cai, Z.J. He, H.Z. Huang, J.L. Long, W.Q. Shen, C. Zhong, J.X. Zuo, Phys. Rev. C74, 064902 (2006)

[32] B.B. Abelev et al. (ALICE), JHEP 06, 190 (2015)

[33] S. Choudhury, D. Sarkar, S. Chattopadhyay, Phys. Rev. C95, 024904 (2017)

[34] C. Alt et al. (NA49), Phys. Rev. C78, 044907 (2008)

[35] C.M. Ko, G.Q. Li, J. Phys. G22, 1673 (1996)

[36] C.M. Ko, V. Koch, G.Q. Li, Ann. Rev. Nucl. Part. Sci. 47, 505 (1997)

[37] G.Q. Li, C.M. Ko, B.A. Li, Phys. Rev. Lett. 74, 235 (1995)

[38] J.L. Ritman et al. (FOPI), Z. Phys. A352, 355 (1995)

[39] S. Pal, C.M. Ko, Z.w. Lin, B. Zhang, Phys. Rev. C62, 061903 (2000)

[40] J. Xu, L.W. Chen, C.M. Ko, Z.W. Lin, Phys. Rev. C85, 041901 (2012)

[41] J. Xu, T. Song, C.M. Ko, F. Li, Phys. Rev. Lett. 112, 012301 (2014)

[42] L. Adamczyk et al. (STAR), Phys. Rev. Lett. 110, 142301 (2013)

[43] M. Gazdzicki, M. Gorenstein, P. Seyboth, Acta Phys. Polon. B42, 307 (2011)

[44] W. Cassing, A. Palmese, P. Moreau, E.L. Bratkovskaya, Phys. Rev. C93, 014902 (2016)

[45] L. McLerran, R.D. Pisarski, Nucl. Phys. A796, 83 (2007)

[46] J. Steinheimer, J. Randrup, Phys. Rev. Lett. 109, 212301 (2012)

[47] F. Li, C.M. Ko, Phys. Rev. C95, 055203 (2017)

[48] K.J. Sun, L.W. Chen, C.M. Ko, Z. Xu, Phys. Lett. B774, 103 (2017)

[49] J. Xu, C.M. Ko, Phys. Lett. B772, 290 (2017)

[50] L. Adamczyk et al. (STAR), Nature 548, 62 (2017)

[51] Z.T. Liang, X.N. Wang, Phys. Rev. Lett. 94, 102301 (2005), [Erratum: Phys. Rev. Lett.96,039901(2006)]

[52] I. Karpenko, F. Becattini, Eur. Phys. J. C 77, 213 (2017)

[53] Y. Xie, D. Wang, L.P. Csernai, Phys. Rev. C 95, 031901 (2017)

[54] L.G. Pang, H. Petersen, Q. Wang, X.N. Wang, Phys. Rev. Lett. 117, 192301 (2016)

[55] Yu.B. Ivanov, A.A. Soldatov, Phys. Rev. C95, 054915 (2017)

[56] H. Li, L.G. Pang, Q. Wang, X.L. Xia, Phys. Rev. C96, 054908 (2017)

[57] Z.W. Lin, C.M. Ko, B.A. Li, B. Zhang, S. Pal, Phys. Rev. C 72, 064901 (2005)

[58] Y. Sun, C.M. Ko, Phys. Rev. C96, 024906 (2017)

[59] S. Cho et al. (ExHIC), Phys. Rev. Lett. 106, 212001 (2011) 
[60] S. Cho et al. (ExHIC), Phys. Rev. C84, 064910 (2011)

[61] S. Cho et al. (ExHIC), Prog. Part. Nucl. Phys. 95, 279 (2017)

[62] R.L. Jaffe, Phys. Rev. Lett. 38, 195 (1977), [Erratum: Phys. Rev. Lett.38,617(1977)]

[63] K. Morita, T. Furumoto, A. Ohnishi, Phys. Rev. C91, 024916 (2015)

[64] L. Adamczyk et al. (STAR), Phys. Rev. Lett. 114, 022301 (2015)

[65] S.R. Beane et al. (NPLQCD), Phys. Rev. Lett. 106, 162001 (2011)

[66] T. Inoue, S. Aoki, T. Doi, T. Hatsuda, Y. Ikeda, N. Ishii, K. Murano, H. Nemura, K. Sasaki (HAL QCD), Nucl. Phys. A881, 28 (2012)

[67] G.A. Miller, Chin. J. Phys. 51, 466 (2013)

[68] Z.Y. Zhang, Y.W. Yu, C.R. Ching, T.H. Ho, Z.D. Lu, Phys. Rev. C61, 065204 (2000)

[69] S. Gongyo et al. (2017), arXiv:1709.00654 [hep-lat]

[70] F. Etminan, H. Nemura, S. Aoki, T. Doi, T. Hatsuda, Y. Ikeda, T. Inoue, N. Ishii, K. Murano, K. Sasaki (HAL QCD), Nucl. Phys. A928, 89 (2014)

[71] K. Morita, A. Ohnishi, F. Etminan, T. Hatsuda, Phys. Rev. C94, 031901 (2016)

[72] T. Hatsuda, K. Morita, A. Ohnishi, K. Sasaki, Nucl. Phys. A967, 856 (2017) 